\title{
Yeni 1H-Pirazol-4-(5H)-il Diazen Türevlerinin Sentezi ve Antimikrobiyal Aktivitelerinin İncelenmesi
}

\author{
Havva KURT ${ }^{1}$, Adnan CETIN² ${ }^{2}$, Sedat BOZARI ${ }^{3}$
}

ÖZET: Bu çalışmada, ilk olarak başlangıç maddesi olan etil 1-(4-bromofenil)-2-(1,3,5-trifenil-1H-pirazol-4-(5H)ilden)hidrazinin (1) sentezi gerçekleştirildi. Elde edilen başlangıç maddesi çeşitli hidrazinlerle etkileştirilerek bir seri yeni 1H-pirazol-4-il diazen (2a-h) türevleri sentezlendi. Sentezlenen bileşikler elementel analiz, IR ve NMR gibi spektral yöntemlerle karakterize edildi. Sentezlenen bileşiklerin antibakteriyel aktiviteleri Gram (+) ve Gram (-) bakteriler üzerinde disk difüzyon yöntemi kullanılarak araştırıldı. Kontrol grubu olarak Gentamisin, Cefotaxime antibiyotikleri kullanılırken sentezlenen bileşiklerin kontrol grubu ile karşılaştırıldığında herhangi bir dozun antibakteriyel aktivite göstermediği gözlendi.

Anahtar Kelimeler: Antimikrobiyal, hidrazin, hidrazon, kontrol grup, pirazol

\section{Antimicrobial Investigation and Synthesis of Novel $1 \mathrm{H}$-Pyrazole-4- (5H)-yl Diazene Derivatives}

\begin{abstract}
In this study, firstly, ethyl 1-(4-bromophenyl)-2-(1,3,5-triphenyl-1H-pyrazol-4-(5H)-ylidene) hydrazine (1), which has starting material, was synthesized. A series of new 1H-pyrazol-4-yl diazene (2a-h) derivatives were synthesized from reaction the obtained starting material and various hydrazines. The synthesized compounds were confirmed by spectral methods such as elemental analysis, IR and NMR. Antibacterial activity of the compounds were investigated by disc diffusion method against to Gram (+) and Gram (-) bacteria. Gentamicin and Cefotaxime antibiotics were used as the control group. None of the doses showed any antibacterial activity compared to the control.
\end{abstract}

Keywords: Antimicrobial, control group, hydrazine, hydrazono, pyrazole

Havva KURT (0000-0002-2951-1934), Muş Alparslan Üniversitesi, Fen Edebiyat Fakültesi, Moleküler Biyoloji Bölümü, Muş, Türkiye Adnan ÇETİN (0000-0001-6487-9489), Muş Alparslan Üniversitesi, Eğitim Fakültesi, Matematik ve Fen Bilimleri Eğitimi, Muş, Türkiye Sedat BOZARI (0000-0001-5265-2236), Muş Alparslan Üniversitesi, Fen Edebiyat Fakültesi, Moleküler Biyoloji Bölümü, Muş, Türkiye Sorumlu yazar/Corresponding Author: Adnan Çetin, adnancetin49@yahoo.com 


\section{GİRIŞ}

Heterosiklik bileşiklerin önemli bir sınıfını oluşturan pirazol türevi bileşikler kayda değer biyolojik aktiviteye sahip olmaları nedeniyle sentetik kimyacılar için son derece önemli hedef moleküllerdir. Çünkü bu moleküllerin göstermiş olduğu kendine has biyolojik aktivite özelliklerinden dolayı pirazol türevlerine olan ilgi günümüzde hızlı bir şekilde artmaktadır. Ayrıca pirazol türevleri antidepresan, anti-piretik, antimikrobiyal, anti-inflammatuar, analjezik, herbisit ve insektisit gibi biyolojik aktivite özelliklerinden dolayı farmasötik kimyada üzerinde en fazla araştırma yapılan bileşik grupları arasında yer almaktadır (Radi, 2010;Hamama et al., 2012;Kaushik et al., 2012;Isloor et al., 2013;Küçükgüzel ve Şenkardeş, 2015;Çetin ve Bildirici, 2016). Aynı zamanda pirazol bileşiklerinin sentezi, sentez yöntemlerinin geliştirilmesi ve biyoaktif özelliklerinin incelenmesi ilaç kimyasında ve sentetik organik kimyada giderek daha fazla önem kazanmaktadır.

Şekil 1'de görüldüğü gibi kanser tedavisinde kullanılan Crizotinib, yaygın bir insektisit olarak kullanılan Fipronil, insomnia tedavisi için kullanılan Zaleplon ve antifungal etkiye sahip Penflufen gibi pirazol yapısı ihtiva eden bu bileşikler ticari satılan pirazollere örnek verilebilir (Hainzl and Casida, 1996; Sanger et al., 1996; Walsh et al., 1998;Adam et al., 2012; Shaw et al., 2013).
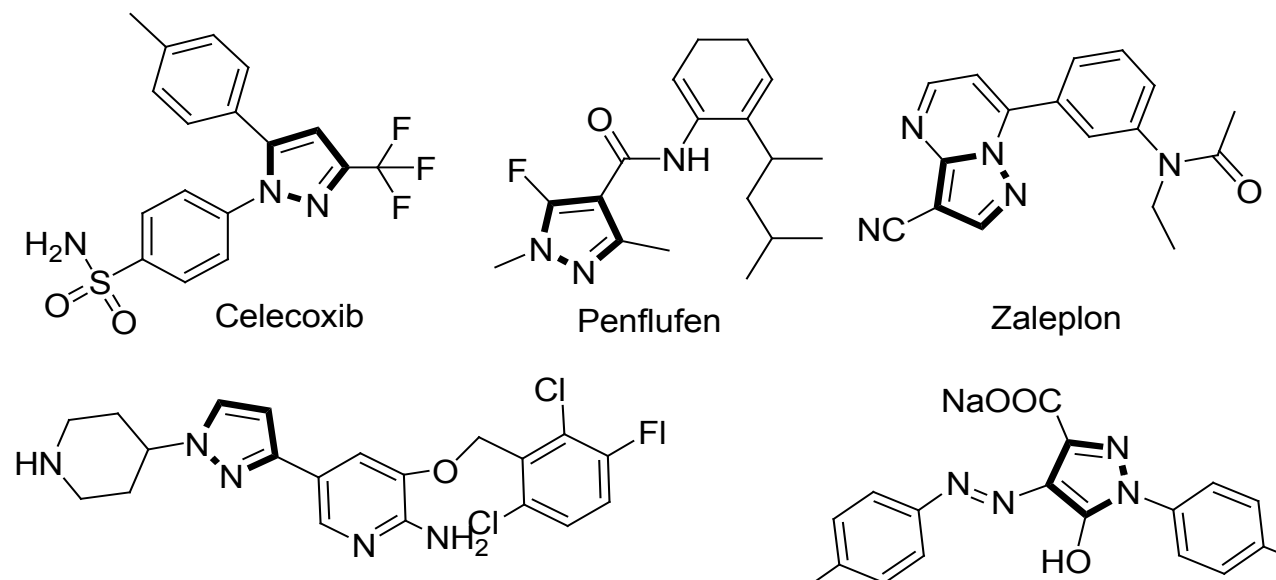

Crizotinib

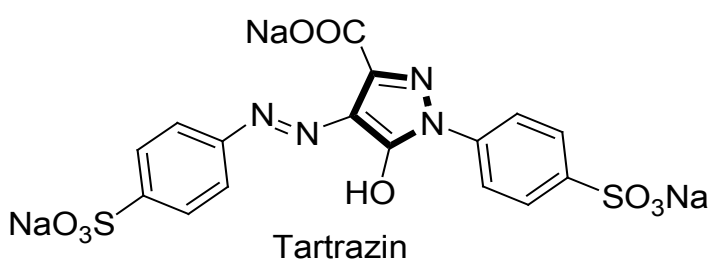

Şekil 1. Bazı önemli sentetik pirazoller

$\mathrm{Bu}$ çalışmalara ek olarak pirazol türevleri birçok alanda başarıyla kullanılmıştır. Örneğin pirazol grubu ihtiva eden polimerler, ışı yayan diyotlar (LED) ve transistor gibi optoelektronik cihazlar için kullanılabilir oldukları görülmüştür (Gondek, 2013;Stakhira et al., 2011;Villafañe et al., 2015; Varghese et al., 2017). $\mathrm{Bu}$ yüzden pirazol yapısı ihtiva eden bileşikler optik sabitleri, optik bant aralığı, kırılma indeksi ve kimyasal özellikler açısından bilimsel ve endüstriyel alanlarda potansiyel kullanımları araştırılmıştır (Zolfigol et al., 2016;Cetin ve ark., 2017;Dohare et al., 2017). Çünkü buna benzer yapılar sahip olduğu optik özelliklerinden dolay1 foto reseptörler, 1şık yayan diyot, güneş pili, ince-film transistor, elektrokromik cihaz, şarj olabilen pil, gibi birçok elektro-optik ve opto-elektronik uygulamalar için potansiyel malzemeler olarak son yıllarda çok yaygın bir şekilde kullanılmaktadır (Gondek, 2013). Diğer bir örnek pirazol yapısı ihtiva eden Tartazin adlı bileşiktir. Bu sentetik bileşik limon sarısı rengi olan bir gida boyasıdır. Ticari olarak gida ve kozmetik sektöründe yaygın olarak kullanılmaktadır (Joule and Mills, 2012). Gida ve boya sanayisinde kullanım alanlarının yaygın olması dünya çapında üzerinde en fazla araştırma yapılan bileşik ve ekonomik önemi en hızlı artan bileşiklerden biri olmasına neden olmuştur. Bizimde sentezlediğimiz bileşikler bu alanda kullanılabilir potansiyele sahip olabilme ihtimali olmas1 çalışmamızın bu yönüyle değerlendirme durumunu ortaya çıkarmıştır. Pirazol türevleri göstermiş oldukları biyolojik aktivitelerden dolayı pestisit ve insektisit olarak tarım endüstrisinde ve ilaç tasarımı olarak kullanıldıklarından eczacılık gibi endüstriyel alanlarda ayrıca fiziksel aktivitelerden dolayı da çok çeşitli teknoloji endüstrisinde geniş bir uygulama alanı bulmaktadır. Bu nedenle bu tür heterosiklik moleküllerin sentezi güncel bir konu olarak araştırmacılar tarafından 
çalışılmaktadır. $\mathrm{Bu}$ moleküllerin elde edilmesi ve çeşitlendirilmesi için geliştirilen yöntemler gün geçtikçe daha fazla önem verilmektedir. Pirazol halkası ihtiva eden bileşiklerin en önemli sentez yöntemlerini kısaca özetlersek; 1,3-dikarbonil, $\alpha, \beta$-doymamış karbonil bileşikleri ve $\beta$-enaminonları gibi bileşikleri içeren uygun hidrazinlerin siklokondensansasyon reaksiyonu ile pirazol eldesi genel olarak yaygın kullanılan bir reaksiyondur (Heller and Natarajan, 2006; Fustero et al., 2011;Çetin, 2016; Radfar et al., 2016). Bu çalışmada, öncelikle başlangıç maddesi soğuk ortamda aşırı asit muamelesiyle diazolama reaksiyonu sonucu substitue 1,3-dikarbonil yapısına sahip 1 bileşiği elde edildi. Sonra çeşitli hidrazinlerle 1 bileşiği uygun ortamda etkileştirilerek substitue pirazol türevlerine dönüştürüldü. Sonra sentezlenen bileşiklerin antibakteriyel aktiflikleri araştırıldı.

\section{MATERYAL VE YÖNTEM}

\section{Kimyasal Maddeler}

Başlangıç maddesi olan etil 1-(4-bromofenill)2-(1,3,5-trifenil-1 H-pirazol-4- $(5 \mathrm{H})$-ilden $)$ hidrazin literatürde gösterildiği gibi elde edilip, yeni bir seri 1H-pirazol-4-il diazen türevleri hazırlanmıştır. Kullanılan başlıca kimyasal maddeler, Merck, Sigma ve Aldrich firmalarından ithal edilen özel reaktifler olup, analitik saflıktadırlar. Reaksiyon ortamında maddelerin tespiti için ince tabaka kromotografisi olarak Merck marka $0.25 \mathrm{~mm}$ silika jel ile kaplı $20 \times 20 \mathrm{~cm}$ çapındaki tabakalar kullanıldı. Erime noktaları Electro termal IA9000 cihazından alınmıştır. İnfrared spektrumları Matson 1000 FT-IR spektrometre cihazı üzerinden alınmıştır. Bant genişlikleri $\mathrm{cm}^{-1}$ cinsinden verilmiştir. Nükleer Magnetik Rezonans $\left({ }^{1} \mathrm{H}-\mathrm{NMR}\right.$ ve ${ }^{13} \mathrm{C}$-NMR) spektrumları Bruker Instrument Avance SeriesSpectrospin DPX-400 Ultra Shield cihazı üzerinden, içerisinde referans miktarda TMS bulunan $\mathrm{CDCl}_{3}$ çözücüsü kullanılarak alınmıştır. Kimyasal kaymalar (ठ) ppm birimi ile verilmiştir.

\section{Bileşiklerin Sentezi}

2-(2-(4-bromofenil)hidrazin)-1,3-difenilpropan1,3-dion (1)

Literatüre göre sentezlendi (Bustos et al., 2011). Verim: $90 \%$, E.N $300{ }^{\circ} \mathrm{C}$

\section{Yeni 1H-Pirazol-5(4H)-on (2a-h) Türevlerinin Sentezi}

50 ml'lik bir balon içerisine çıkış bileşiği $1(0.1 \mathrm{~g}$, $0.25 \mathrm{mmol})$ ve sülfürik asid $(0,75 \mathrm{mmol})$ etanol $(5 \mathrm{ml})$ içinde çözüldü. Karışıma etanol ( $5 \mathrm{ml})$ içinde çözülmüş uygun hidrazinler $(0.25 \mathrm{mmol})$ (hidrazin hidrat, metil hidrazin, etil hidrazin, fenil hidrazin, 2,5-dimetil fenil hidrazin, 3,4-dimetil fenil hidrazin ve 2-nitrofenil hidrazin) damla damla eklendi. Karışım kaynama sıcaklığında 4 saat 1 sitıldı. Karışım oda sıcaklığında kendi halinde soğumaya bırakıldı. Bir süre sonra oluşan çökelek süzülüp kurutuldu. Ürünler etanolde kristallendirilerek saflaştırıldı.

1-(4-bromofenil)-2-(3,5-difenil-4H-pirazol4iliden)hidrazin (2a)

1 ve hidrazin hidrat kullanıld1. 2a genel prosedüre göre sentezlendi. Verim: $0.09 \mathrm{~g}, 80 \%$. EN $165-167^{\circ} \mathrm{C}$, ${ }^{1} \mathrm{H}-\mathrm{NMR}\left(400 \mathrm{MHz}, \mathrm{CDCl}_{3}\right) \delta(\mathrm{ppm}): 8.8($ br.s, $1 \mathrm{H}$, -NH), 8.0-6.5 (m, 14H, Ar-H). ${ }^{13} \mathrm{C}-\mathrm{NMR}(100 \mathrm{MHz}$, $\left.\mathrm{CDCl}_{3}\right) \delta(\mathrm{ppm}): 161.2,158.2,146.4,142.1,137.8$, $132.5,131.1,130.5,129.6,129.2,128.7,128.4,121.4$, 109.6. IR ( $\left(\mathrm{cm}^{-1}\right): 3142,3062,2921,1610,1496$, 1444, 1316. Hesaplanan $\mathrm{C}_{21} \mathrm{H}_{15} \mathrm{BrN}_{4}(403.27 \mathrm{~g} / \mathrm{mol}): \mathrm{C}$, 62.54; H, 3.75; N, 13.89. Bulunan: C, 62.60; H, 3.71; $\mathrm{N}, 13.82$.

1-(4-bromofenil)-2-(1-metil-3,5-difenil-1 Hpirazol-4-il)diazen (2b)

1 ve metil hidrazin kullanıldı. 2b genel prosedüre göre sentezlendi. Verim: 0.08 g, $72 \%$. E.N 162-164 ${ }^{\circ} \mathrm{C}$, ${ }^{1} \mathrm{H}-\mathrm{NMR}\left(400 \mathrm{MHz}, \mathrm{CDCl}_{3}\right) \delta$ (ppm): 8.1-6.6 (m, 14H, Ar-H), 1.9 (s, 3H, - $\left.\mathrm{CH}_{3}\right) \cdot{ }^{13} \mathrm{C}-\mathrm{NMR}(100 \mathrm{MHz}$, $\left.\mathrm{CDCl}_{3}\right) \delta$ (ppm): 160.0, 157.1, 142.3, 138.4, 133.1, 130.5, 129.6, 129.5, 129.4, 128.7, 128.3, 123.2, 110.5, 32.1. IR ( $\left(v, \mathrm{~cm}^{-1}\right): 3060,2862,1598,1498,1442,1325$. Hesaplanan $\mathrm{C}_{22} \mathrm{H}_{17} \mathrm{BrN}_{4}(417.32 \mathrm{~g} / \mathrm{mol}): \mathrm{C}, 63.32 ; \mathrm{H}$, 4.11; N, 13.43. Bulunan: C, 63.31; H, 4.15; N, 13.39 .

1-(4-bromofenil)-2-(1-etil-3,5-difenil-1 Hpirazol-4-il)diazen (2c)

1 ve etil hidrazin kullanıldı. 2c genel prosedüre göre sentezlendi. Verim: $0.09 \mathrm{~g}, 78 \%$. EN $171-173{ }^{\circ} \mathrm{C}$, ${ }^{1} \mathrm{H}-\mathrm{NMR}\left(400 \mathrm{MHz}, \mathrm{CDCl}_{3}\right)$ : $\delta$ 7.9-6.4 (m, 14H, Ar-H), $3.3\left(\mathrm{~m}, 2 \mathrm{H},-\mathrm{CH}_{2}\right), 1.4\left(\mathrm{t}, 3 \mathrm{H},-\mathrm{CH}_{3}\right) \cdot{ }^{13} \mathrm{C}-\mathrm{NMR}(100$ $\left.\mathrm{MHz}, \mathrm{CDCl}_{3}\right): \delta 152.5,142.7,136.4,133.0,131.9,130.7$, 130.3, 129.7, 129.6, 129.4, 128.1, 123.0, 122.3, 110.6, 36.4, 15.9. IR ( $\left(v, \mathrm{~cm}^{-1}\right): 3064,2875,2221,1609,1498$, 1444, 1316. Hesaplanan $\mathrm{C}_{23} \mathrm{H}_{21} \mathrm{BrN}_{4}(431.34 \mathrm{~g} / \mathrm{mol})$ : 
C, 63.75; H, 4.88; N, 12.93. Bulunan: C, 63.79; H, 4.89; $\mathrm{N}, 12.92$.

\section{1-(4-bromofenil)-2-(1,3,5-trifenil-1H-pirazol-4- il)diazen (2d)}

1 ve fenil hidrazin kullanıldı. 2d genel prosedüre göre sentezlendi. Verim: $0.08 \mathrm{~g}, 66 \%$. EN 181-184 ${ }^{\circ} \mathrm{C},{ }^{1} \mathrm{H}-\mathrm{NMR}\left(400 \mathrm{MHz}, \mathrm{CDCl}_{3}\right): \delta 8.0-6.3(\mathrm{~m}, 19 \mathrm{H}$, Ar-H). ${ }^{13} \mathrm{C}-\mathrm{NMR}\left(100 \mathrm{MHz}, \mathrm{CDCl}_{3}\right): \delta 150.3,143.4$, $141.0,136.2,133.4,132.2,131.2,130.5,130.2,129.9$, 129.3, 128.9, 128.4, 128.2, 127.5, 122.0, 109.7. IR ( $v$, $\left.\mathrm{cm}^{-1}\right): 3064,2871,1606,1497,1442,1321$. Hesaplanan $\mathrm{C}_{27} \mathrm{H}_{19} \mathrm{BrN}_{4}(479.38 \mathrm{~g} / \mathrm{mol}): \mathrm{C}, 67.65 ; \mathrm{H}, 4.00 ; \mathrm{N}, 11.69$. Bulunan: C, 67.63; H, 4.03; N, 11.67.

1-(4-bromofenil)-2-(1-(2,5-dimetilfenil)-3,5difenil-1H-pirazol-4-il)diazen (2e)

1 ve 2,5-dimetilfenil hidrazin kullanıldı. 2e genel prosedüre göre sentezlendi. Verim: $0.1 \mathrm{~g}, 70 \%$. EN 179-181 ${ }^{\circ} \mathrm{C},{ }^{1} \mathrm{H}-\mathrm{NMR}\left(400 \mathrm{MHz}, \mathrm{CDCl}_{3}\right.$ ): $\delta$ 8.1-6.5 (m, 17H, Ar-H), $2.0\left(\mathrm{~s}, 3 \mathrm{H},-\mathrm{CH}_{3}\right), 1.5\left(\mathrm{~s}, 3 \mathrm{H},-\mathrm{CH}_{3}\right)$. ${ }^{13} \mathrm{C}-\mathrm{NMR}\left(100 \mathrm{MHz}, \mathrm{CDCl}_{3}\right): \delta 151.1,145.1,142.0$, 136.2, 134.6, 131.8, 130.5, 129.6, 129.4, 129.2, 129.1, $127.9,127.8,127.7,127.3,121.1,112.1,22.1,16.7$. IR $\left(v, \mathrm{~cm}^{-1}\right): 3062,2856,1601,1497,1442,1324$. Hesaplanan $\mathrm{C}_{29} \mathrm{H}_{23} \mathrm{BrN}_{4}(507.43 \mathrm{~g} / \mathrm{mol}): \mathrm{C}, 68.64 ; \mathrm{H}$, 4.57; N, 11.04. Bulunan: C, 68.65; H, 4.52; N, 11.03 .

1-(4-bromofenil)-2-(1-(3,4-dimetilfenil)-3,5difenil-1H-pirazol-4-il)diazen (2f)

1 ve 3,4-dimetilfenil hidrazin kullanıldı. $2 \mathrm{f}$ genel prosedüre göre sentezlendi. Verim: $0.09 \mathrm{~g}, 72 \%$. EN 182-184 ${ }^{\circ} \mathrm{C},{ }^{1} \mathrm{H}-\mathrm{NMR}\left(400 \mathrm{MHz}, \mathrm{CDCl}_{3}\right): \delta 8.1-6.3$ $(\mathrm{m}, 17 \mathrm{H}, \mathrm{Ar}-\mathrm{H}), 1.9\left(\mathrm{~s}, 3 \mathrm{H},-\mathrm{CH}_{3}\right), 1.6\left(\mathrm{~s}, 3 \mathrm{H},-\mathrm{CH}_{3}\right)$. ${ }^{13} \mathrm{C}-\mathrm{NMR}\left(100 \mathrm{MHz}, \mathrm{CDCl}_{3}\right): \delta 156.3,144.2,137.5$, 131.3, 131.0, 130.4, 129.9, 129.6, 129.2, 129.1, 128.8, 128.2, 126.1, 121.9, 109.8, 21.6, 17.1. IR $\left(v, \mathrm{~cm}^{-1}\right): 3066$, 2871, 1603, 1498, 1442, 1317. Hesaplanan $\mathrm{C}_{29} \mathrm{H}_{23} \mathrm{BrN}_{4}$ (507.42 g/mol): C, 68.64; H, 4.57; N, 11.04. Bulunan: C, 68.66; H, 4.59; N, 11.01 .

1-(4-bromofenil)-2-(1-(4-bromofenil)-3,5difenil-1H-pirazol-4-il)diazen (2g)

1 ve 4-bromo fenil hidrazin kullanıldı. 2g genel prosedüre göre sentezlendi. Verim: $0.11 \mathrm{~g}, 75 \%$. EN 202-204 ${ }^{\circ} \mathrm{C},{ }^{1} \mathrm{H}-\mathrm{NMR}\left(400 \mathrm{MHz}, \mathrm{CDCl}_{3}\right.$ ): $\delta \quad 8.1-6.5$ (m, 18H, Ar-H). ${ }^{13} \mathrm{C}-\mathrm{NMR}\left(100 \mathrm{MHz}, \mathrm{CDCl}_{3}\right): \delta 155.7$, 146.4, 142.8, 139.5, 131.2, 130.8, 130.7, 129.7, 129.6,
129.5, 129.3, 128.5, 127.7, 121.7, 122.2, 109.3. IR ( $v$, $\left.\mathrm{cm}^{-1}\right): 3062,2864,1609,1497,1444,1323$. Hesaplanan $\mathrm{C}_{27} \mathrm{H}_{18} \mathrm{Br}_{2} \mathrm{~N}_{4}(558.28 \mathrm{~g} / \mathrm{mol}): \mathrm{C}, 58.09 ; \mathrm{H}, 3.25 ; \mathrm{N}$, 10.04. Bulunan: C, 58.03; H, 3.24; N, 10.02 .

1-(4-bromofenil)-2-(1-(2-nitrofenil)-3,5-difenil1H-pirazol-4-il)diazen (2h)

1 ve 2-nitro fenil hidrazin kullanıldı. 2 h genel prosedüre göre sentezlendi. Verim: $0.09 \mathrm{~g}, 66 \%$. EN 199-201 ${ }^{\circ} \mathrm{C}$, ${ }^{1} \mathrm{H}-\mathrm{NMR}\left(400 \mathrm{MHz}, \mathrm{CDCl}_{3}\right): \delta 8.0-6.3$ (m, 18H, Ar-H). ${ }^{13} \mathrm{C}-\mathrm{NMR}\left(100 \mathrm{MHz}, \mathrm{CDCl}_{3}\right): \delta 159.6$, 144.1, 141.5, 139.3, 133.1, 132.4, 131.7, 131.6, 131.2, $130.1,130.0,129.9,129.8,129.1,128.8,126.8,122.3$, 121.4, 109.8. IR $\left(v, \mathrm{~cm}^{-1}\right): 3065,2871,1604,1496$, 1442,1315 . Hesaplanan $\mathrm{C}_{27} \mathrm{H}_{18} \mathrm{Br}_{2} \mathrm{~N}_{5} \mathrm{O}(524.38 \mathrm{~g} / \mathrm{mol})$ : C, 61.84; H, 3.46; N, 13.36. Bulunan: C, 61.86; H, 3.45; $\mathrm{N}, 13.34$.

\section{Çalışmada Kullanılan Mikroorganizmalar}

Sentezlenen bileşiğin anti mikrobiyal aktivitesini ölçmek amaciyla Klebsiella pneumoniae ATCC 13883, Esherichia coli ATCC 25922, Pseudomonas aeruginosa ATCC27853, Enterobacter aerogenes ATCC 13048, Bacillus megatarium ATCC 14581, Staphylococcus aureus ATCC 29213 ve Bacillus subtilis ATCC 6633 bakterileri kullanıldı.

\section{Antibakteriyel Aktivite Ölçümü}

Sentezlenen bileşikler yukarıda bahsedilen mikroorganizmalara karşı muhtemel antimikrobiyal aktivitesi disk difüzyon yöntemiyle belirlendi. Pozitif kontrol olarak Gentamisin ve Cefotaxime antibiyotik diskleri kullanıldı (Zaidan et al., 2005). Sentezlenen maddeler, \%10'luk DMSO'da çözülerek altı (0.03125, $0.0625,0.125,0.25,0.5$ ve $1 \mathrm{mg} / \mu \mathrm{l})$ farklı dozda hazırlanmış $10 \mathrm{~mm}$ 'lik steril Oxoid disklere $10 \mu \mathrm{l}$ yüklenerek bakterilere uygulandı. Mikroorganizmalar uygun besi yeri ve sicaklıkta bir gece etüvde bekletildikten sonra oluşan zonlar ölçüldü.

\section{BULGULAR VE TARTIŞMA}

Substitüe pirazollerin sentezinin ilk aşamasında, 2-(2-(4-bromofenil)hidrazin)-1,3-difenilpropan-1,3dion (1) bileşiği literatüre göre sentezlendi (Bustos et al., 2011). Başlangıç bileşiği olan 1 1,3-difenilpropan1,3-dion ve 4-bromoanilinin soğuk ortamda diazolama reaksiyonu sonucu elde edildi (Şekil 2). 


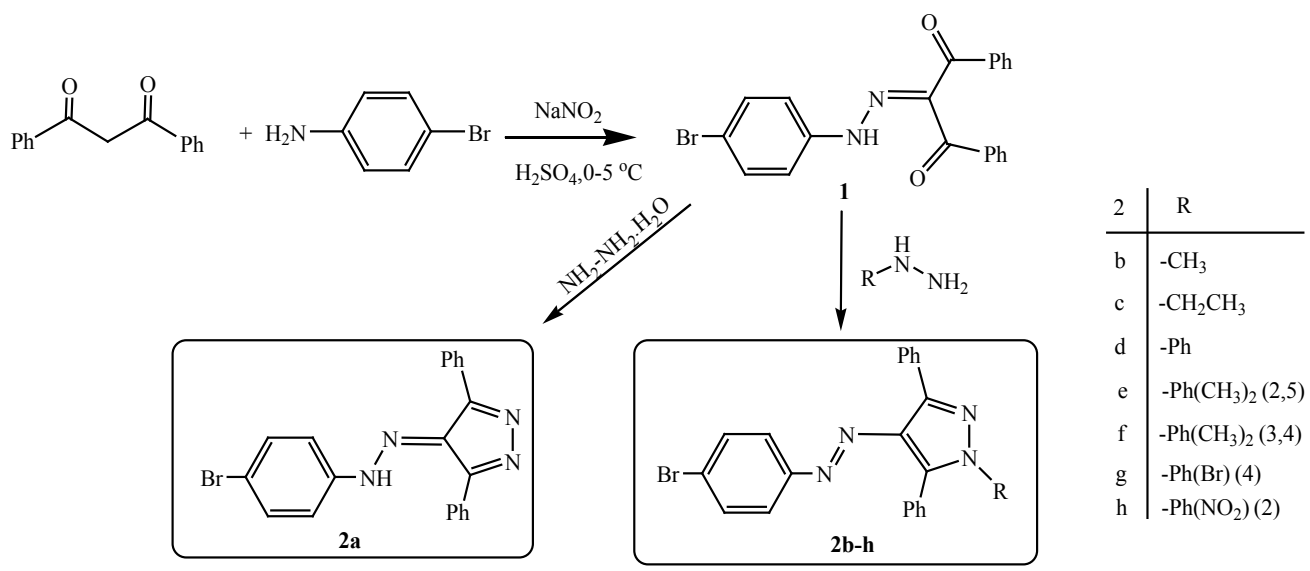

Şekil 2. 1 ve 2a-h bileşiklerinin sentezi

Çıkış bileşiği sentezlendikten sonra hidrazinlerin kondensazyon reaksiyonu ile tepkimeye girmesi sonucu 1H-pirazol-4(5H)-iliden (2a-h) bileşikleri elde edildi. Yeni sentezlenen bileşikler için kullanılan hidrazinler ve elde edilen ürünlerin verimleri Çizelge 1 'de verilmiştir.

Çizelge 1. Sentezde kullanılan hidrazinler, elde edilen ürünler, erime noktaları ve verimleri

Bileşik Hidrazin


Uygun reaksiyon şartlarında $\mathbf{1}$ ve çeşitli hidrazinlerin halkalaşma reaksiyonu sonucu elde edilen $1 H$-pirazol-4(5H)-iliden (2a-h) bileşiklerinin verimleri ortalama \% 66-80 arasındadır. 2a-h bileșiklerin yap1 tayinleri esas olarak IR, ${ }^{1} \mathrm{H}$ NMR ve ${ }^{13} \mathrm{C}$ NMR spektroskopi cihazlarına dayanmaktadır. Elde edilen yeni 2a'nın IR spektrumuna bakıldığında $3142 \mathrm{~cm}^{-1}$ gözlenen pik -NH bağ gerilme titreşiminden, $3060 \mathrm{~cm}^{-1}$ aromatik halkanın $-\mathrm{CH}$ titreşimleri ve $1610 \mathrm{~cm}^{-1}-\mathrm{C}=\mathrm{N}$ imin gözlenen titreşim sinyallerinden kaynaklandığ 1 söylenebilir. ${ }^{13} \mathrm{C}$ NMR spektrumu incelendiğinde $\delta=$ $161.2(\mathrm{C}=\mathrm{N}$ veya $\mathrm{C}-4), 150.2(\mathrm{C}-3)$ ve $146.4(\mathrm{C}-5)$ ppm de görülen sinyallerin pirazol halkasındaki karbon atomlarına işaret ettiği ve ${ }^{1} \mathrm{H}$ NMR spektrumuna bakıldığında ise $\delta=8.1 \mathrm{ppm}$ de gözlenen sinyallerin $-\mathrm{NH}$ protonlarından kaynaklandığı söylenebilir. Sentezlenen yeni $\mathbf{2 b}$-h bileşiklerinin IR spektrumuna bakıldığında sirasıyla 3226-3124 $\mathrm{cm}^{-1}$ arasında $-\mathrm{NH}$ titreşimlerinin, $1598-1552 \mathrm{~cm}^{-1}$ arasinda aromatik halkadaki $(\mathrm{C}=\mathrm{C})$ titreşim sinyallerinin ve $3082-3060 \mathrm{~cm}^{-1}$ arasında ise aromatik $(-\mathrm{CH})$ protonlarının sinyallerinin olduğu görülmektedir. Karakteristik ${ }^{13} \mathrm{C}$ NMR spektrumu incelendiğinde pirazol halkasındaki 159.6-152.3 ppm arasinda (C-3), 112.1-109.4 ppm arasinda (C-4) ve 147.2-143.4 ppm arasında (C-5) karbonlarının pikleri görülmektedir. Tüm sentezlenen bileşiklerin spektral verileri detaylı şekilde gözden geçirildiğinde ön

\section{KAYNAKLAR}

Adam NM, Wilson SP, Duvert P, 2012. The development of penflufen as a seed treatment for potatoes in northern Europe. In The Dundee Conference. Crop Protection in Northern Britain, 28-29 February 2012, Cambridge.

Bustos C, Alvarez-Thon L, Cárcamo JG, Garland MT, Sánchez C, 2011. 2-[2-(4-Bromophenyl)hydrazinylidene]-1,3diphenylpropane-1,3-dione, Acta Crystallographica E 67: 1426-1431.

Cetin A, Gündüz B, Menges N, Bildirici I, 2017. Unsymmetrical pyrazole-based new semiconductor oligomer: synthesis and optical properties, Polymer Bulletin 74: 2593-2604.

Çetin A, 2016. Synthesis of pyrazoles via electrophilic cyclization of alkynes containing thiophene, Letters in Organic Chemistry 13: $310-315$

Çetin A, Bildirici İ, 2018. A study on synthesis and antimicrobial activity of 4-acyl-pyrazoles, Journal of Saudi Chemical Society, 22:279-296

Dohare P, Ansari KR, Quraishi MA, Obot IB, 2017. Pyranpyrazole derivatives as novel corrosion inhibitors for mild steel useful for industrial pickling process: Experimental and Quantum Chemical study, Journal of Industrial and Engineering Chemistry 52: 197-210. görülen yapılar ile sonuçların hesaplanan değerlerle uyum içinde olduğu gözlendi (Çetin ve Bildirici, 2016). Sentezlenen bileşiklerin DMSO'da çözünen altı farklı dozda uygulandı $\breve{g} 1$ ve farklı mikroorganizmalara karşı herhangi bir antibakteriyel aktivite göstermediği belirlendi.

\section{SONUC}

Sonuç olarak, ilk defa tarafımızca sentezlenen pirazol türevleri ortalama verimlerle elde edildi. Sentezlenen bileşikler spektroskopik yöntemlerle yapıları aydınlatıldı. Sentezlenen bileşiklerin antibakteriyel aktiflikleri incelendi ve biyolojik aktivite göstermediği bulunmuştur. Ancak literatürde buna benzer bileşiklerin gıda alanında, endüstriyel ve sanayi alanlarında kullanıldıkları görülmüştür. Bizim çalışmada elde edilen bileşikler bu alanlarda aktivite gösterebilecek potansiyele sahip olma özelliğiyle önemli olacağı düşüncesindeyiz.

\section{TEŞEKKÜR}

Muş Alparslan Üniversitesi Bilimsel Araştırma Projeleri Başkanlığı'na (Proje no: MSÜ15-EMF-G05) çalışmamıza sağladığı maddi destekten dolayı teşekkür ederiz.

Fustero S, Sánchez-Roselló M, Barrio P, Simón-Fuentes A, 2011. From 2000 to Mid-2010: a fruitful decade for the synthesis of pyrazoles, Chemical Reviews 111: 6984-7034.

Gondek E, 2013. Photovoltaic solar cells based on pyrazole derivative, Materials Letter 112: 94-99.

Hainzl D, Casida JE, 1996. Fipronil insecticide: novel photochemical desulfinylation with retention of neurotoxicity, Proceedings of the National Academy of Sciences 93: 12764-12767.

Hamama WS, El-Gohary HG, Soliman M, Zoorob HHA, 2012. Versatile synthesis, pm3-semiempirical, antibacterial and antitumor evaluation of some bioactive pyrazoles, Journal Heterocyclic Chemistry 49: 543-554.

Heller ST, Natarajan SR. 2006. 1,3-Diketones from acid chlorides and ketones: a rapid and general one-pot synthesis of pyrazoles, Organic Letters 8: 2675-2678.

Isloor AM, Vijesh AM, Shetty P, Sundershan S, Fun HK, 2013. New pyrazole derivatives containing 1, 2, 4-triazoles and benzoxazoles as potent antimicrobial and analgesic agents, European Journal Medicinal Chemistry 62: 410-415.

Joule JA, Mills K, 2012. Heterocyclic chemistry at a glance. Third Edition, London, UK. 230 p.

Kaushik D, Kumar R, Khan SA, Chawla G, 2012. Pharmacological screening for anti-inflammatory, analgesic activity of pyrazolyl 
derivatives along with molecular docking studies. Med Chem Res 21: 3646-3655.

Küçükgüzel ŞG, Şenkardeş S, 2015. Recent advances in bioactive pyrazoles. Euro J Med Chem 97: 786-815.

Radi S, Salhi S, Radi A, 2010. Synthesis and preliminary biological activity of some new pyrazole derivatives as acyclonucleoside analogues. Lett Drug Des Discov 7: 27-30.

Sanger DJ, Morel E, Perrault G, 1996. Comparison of the pharmacological profiles of the hypnotic drugs, zaleplon and zolpidem. Euro J Pharma 313: 35-42.

Shaw AT, Kim DW, Nakagawa K, Seto T, Crinó L, Ahn MJ, Wu Y L, 2013. Crizotinib versus chemotherapy in advanced ALKpositive lung cancer, New England Journal of Medicine, 368: 2385-2394.

Stakhira P, Cherpak V, Khomyak S, Volynyuk D, Hotra Z, Voznyak L, Oliynyk O, 2011. Properties of 2, 6-di-tert-butyl-4-(2, 5-diphenyl-3, 4-dihydro-2H-pyrazol-3-yl)-phenol as holetransport material for life extension of organic light emitting diodes. Opt Mater 33: 1727-173.

Varghese B, Al-Busafi SN, Suliman FO, Al-Kindy SM, 2017. Unveiling a versatile heterocycle: pyrazoline-a review, RSC Advances, 7: 46999-47016.
Villafañe F, Gómez-Iglesias P, Guyon F, Khatyr A, Ulrich G, Knorr M, Martín-Alvarez JM, 2015. Luminescent rhenium (I) tricarbonyl complexes with pyrazolylamidino ligands: photophysical, electrochemical, and computational studies. Dalton Transactions 44: 17516-17528.

Walsh JK, Fry J, Erwin CW, Scharf M, Roth T, Vogel GW, 1998. Efficacy and tolerability of 14-day administration of zaleplon $5 \mathrm{mg}$ and $10 \mathrm{mg}$ for the treatment of primary insomnia, Clinical Drug Investigation 16: 347-354.

Zaidan MR, Noor Rain A, Badrul AR, Adlin A, Norazah A, Zakiah I. 2005. In vitro screening of five local medicinal plants for antibacterial activity using disc diffusion method. Trop Biomed 22: 165-170.

Zolfigol MA, Ayazi-Nasrabadi R, Baghery S, Khakyzadeh V, Azizian S, 2016. Applications of a novel nano magnetic catalyst in the synthesis of 1, 8-dioxo-octahydroxanthene and dihydropyrano [2,3-c] pyrazole derivatives, Journal of Molecular Catalysis A: Chemical 418: 54-67. 